\title{
Childhood socioeconomic deprivation, but not current mood, is associated with behavioural disinhibition in adults
}

Tünde Paál, Thomas Carpenter, Daniel Nettle

There is evidence to suggest that impulsivity is predicted by socioeconomic background, with people from more deprived backgrounds tending to be more impulsive, and by current mood, with poorer mood associated with greater impulsivity. However, impulsivity is not a unitary construct, and previous research in this area has focused on measures of 'waiting' impulsivity rather than behavioural disinhibition. We administered a standard measure of behavioural disinhibition, the stop-signal task, to 58 adult participants from a community sample. We had measured socioeconomic background using participant postcode at age 16, and assigned participants to receive either a neutral or a negative mood induction. We found no effects of mood on behavioural disinhibition, but we found a significant effect of socioeconomic background. Participants who had lived in more deprived postcodes at age 16 showed longer stop-signal reaction times, and hence greater behavioural disinhibition. The pattern was independent of participant age and overall reaction time. Though caution is required inferring causality from correlation, it is possible that that experiencing socioeconomic deprivation in childhood and adolescence may lead to greater behavioural disinhibition in adulthood. 
2 Childhood socioeconomic deprivation, but not current mood, is associated with behavioural disinhibition in 3 adults

4

5

6 Tünde Paál1,2

$7 \quad$ Thomas Carpenter ${ }^{1}$

8 Daniel Nettle ${ }^{1 *}$

9

10 1. Centre for Behaviour and Evolution \& Institute of Neuroscience, Newcastle University, Newcastle, UK

112 2. University of Pécs, Institute of Psychology, Pécs, Hungary

12

13

14

15

16

$17 *$ To whom correspondence should be addressed: daniel.nettle@ncl.ac.uk

18

19

20

21 Author note: The first two authors contributed equally to this paper.

22 


\section{ABSTRACT}

24 There is evidence to suggest that impulsivity is predicted by socioeconomic background, with people from

25 more deprived backgrounds tending to be more impulsive, and by current mood, with poorer mood associated 26 with greater impulsivity. However, impulsivity is not a unitary construct, and previous research in this area has

27 focused on measures of 'waiting' impulsivity rather than behavioural disinhibition. We administered a standard 28 measure of behavioural disinhibition, the stop-signal task, to 58 adult participants from a community sample.

29 We had measured socioeconomic background using participant postcode at age 16, and assigned participants

30 to receive either a neutral or a negative mood induction. We found no effects of mood on behavioural

31 disinhibition, but we found a significant effect of socioeconomic background. Participants who had lived in

32 more deprived postcodes at age 16 showed longer stop-signal reaction times, and hence greater behavioural

33 disinhibition. The pattern was independent of participant age and overall reaction time. Though caution is

34 required inferring causality from correlation, it is possible that that experiencing socioeconomic deprivation in 35 childhood and adolescence may lead to greater behavioural disinhibition in adulthood.

36

37 
40 Impulsivity is an important psychological construct, because it has been linked to a number of outcomes that

41 are problematic from both individual and societal perspectives, such as addiction and criminal behaviour (Pratt

42 \& Cullen, 2000; Perry \& Carroll, 2008; Moffitt et al., 2011; Sharma, Markon \& Clark, 2014). Individual

43 differences in impulsivity are likely to reflect multiple influences, from genetics (Kreek et al., 2005), to early

44 developmental factors (Pettersson et al., 2014), to aspects of the immediate social context (Kidd, Palmeri \&

45 Aslin, 2013). Here, we focus on two factors that have recently been shown to be associated with impulsivity:

46 socioeconomic position and negative mood. A substantial number of studies have presented evidence that individuals of lower socioeconomic position tend to be more impulsive than those of higher socioeconomic position (Lawrance, 1991; Green et al., 1996; Adams \& White, 2009). Though causality is difficult to establish definitively, the relationship has been argued to be at least partly causal (Haushofer \& Fehr, 2014). That is, experiencing socioeconomic hardship may make people become more impulsive, rather than, for example, more impulsive people being downwardly economically mobile. Other studies have shown that negative mood can also make people more impulsive (Lerner, Li \& Weber, 2012). Here, the causality is much easier to demonstrate, since the negative mood was induced experimentally through watching a film clip about a death (or a neutral control film clip) prior to the impulsivity task. The socioeconomic and mood effects on impulsivity may well be linked; part of the reason that people of lower socioeconomic position are characterised as more impulsive may be that their mood is more negative much of the time (Haushofer \& Fehr, 2014). However, whether there is also a 'mood-independent' effect of socioeconomic position on impulsivity is not at present well understood.

Impulsivity, however, is not a unitary construct (Reynolds et al., 2006; Sharma, Markon \& Clark, 2014; Stahl et al., 2014). Although a number of typologies of impulsivity have been proposed, a common distinction is between the unwillingness to wait for a deferred outcome ('waiting impulsivity' or impulsive choice) and the inability to stop oneself from making a response that has been cued or initiated by the context ('stopping impulsivity', impulsive action, or behavioural disinhibition) (Reynolds et al., 2006; Perry \& Carroll, 2008; Brevers et al., 2012). Although both types are often referred to as impulsivity in the literature, measures of waiting impulsivity do not tend to be substantially correlated with measures of behavioural disinhibition (Reynolds et al., 2006). Almost all of the evidence on socioeconomic position and current mood as predictors of impulsivity comes from waiting impulsivity tasks. For behavioural disinhibition, one study found no socioeconomic patterning in a large community sample of children and adolescents (Crosbie et al., 2013). Beyond this, there is little research looking for a socioeconomic gradient, and there have been no experimental studies 
manipulating mood to examine the consequences for behavioural disinhibition. In this study, we therefore administered a standard measure of behavioural disinhibition, the stop-signal task, to adults from a community sample, having first measured their socioeconomic background, and randomly assigned them to receive either a negative mood induction or a control procedure.

74 The stop-signal task measures behavioural inhibition by presenting participants with two concurrent tasks, the go task and the stop task. The go task is a visual identification task to be completed as rapidly as possible. The stop task involves presentation of an additional cue (the stop signal) that tells the participant not to complete the go task on that particular trial. The stop signal occurs on only a minority of trials. Thus, the participant forms a prepotent response pattern of completing the go task on every trial, but on a minority of trials has to inhibit that prepotent response. Whether she successfully inhibits the response depends on which process completes first: the prepotent impulse to go, or the inhibitory impulse produced in response to the stop signal (Logan \& Cowan, 1984; Logan, Cowan \& Davis, 1984). The temporal offset between the appearance of the gosignal and the appearance of the stop-signal is varied in order to identify, for each participant, the offset at which the stop impulse completes sooner than the go impulse more often than not. This makes it possible to assign each participant a stop-signal reaction time (SSRT). This is in effect a measure of how long it takes a person to mount an inhibitory response to a prepotent action impulse. A longer SSRT equates to poorer behavioural inhibition (or equivalently, greater behavioural disinhibition). SSRT scores have been validated as measures of stopping impulsivity in studies of drug addiction (Fillmore et al., 2002). When analysing SSRTs, it is important to control for participants' average reaction time on the go trials (the go reaction time, or GRT), since some of the variance in SSRT may be explained by how slow the go response is rather than how fast the inhibitory response is. Both SSRT and GRT have also been shown to increase with age in adulthood (Williams et al., 1999; Bedard et al., 2010).

There are multiple ways of conceptualizing and assessing socioeconomic position. Recent studies have suggested that exposure to deprived neighbourhoods across childhood may be a key predictor of psychological outcomes (Sampson, Sharkey \& Raudenbush, 2008; Sastry \& Pebley, 2010; Sharkey \& Elwert, 2011). We thus chose to focus on neighbourhood socioeconomic deprivation rather than individual socioeconomic status. We therefore decided to use residential postcodes to obtain a deprivation score for the neighbourhood in which the participant had grown up. The UK has high-quality publicly-available neighbourhood deprivation data resolved to a small spatial scale, based on the average of indices across multiple domains of deprivation. 
101 emotionally neutral, one aimed to produce a depression-like negative mood, and one aimed to produce an

102 elated positive mood. The Velten statements have been widely used, and previous studies have shown that 103 participants' self-rated mood is more depressed and more anxious following the negative than the control set

104 (Kenealy, 1986; Westermann, Stahl \& Hesse, 1996). Thus, the effect of the manipulation appears to be both a 105 reduction in positive affect and an increase in negative affect (Watson \& Tellegen, 1985). Although many 106 studies have found significant effects of the Velten mood induction procedure on mood, it is important to 107 include a manipulation check to verify this in each experiment, since a null finding in relation to the outcome of 108 interest is uninterpretable unless the mood manipulation is known to have worked (Kenealy, 1986).

109 Our predictions were that participants from more deprived backgrounds, and participants assigned to the 110 negative condition, would show relatively longer SSRTs, once appropriate control was made for age and GRT.

111 We noted the possibility that there might also be interactions between socioeconomic background and mood 112 condition, since some recent studies have suggested that people from different socioeconomic backgrounds 113 react differently to cues of current adversity (Griskevicius et al., 2013).

\section{METHODS}

\section{Ethics and Participants}

116 The study was authorised by the Newcastle University Faculty of Medical Sciences Ethics Committee under 117 approval number 00655/2013. Participants were an opportunity sample of individuals who had grown up in 118 the UK, recruited by means of the Institute of Neuroscience participant pool, Newcastle University. This is a 119 large database of email addresses of people who have shown an interest in taking part in neuroscience or medical research. It includes students, staff of the university, and other residents of the city, and is thus reasonably diverse in terms of ages and socioeconomic backgrounds. A compensation of $£ 5$ was offered in exchange for participation. The data includes two samples gathered in separate years by Thomas Carpenter and Tünde Paál respectively. All procedures were identical in the two sub-samples. We have repeated all analyses in this paper with experimenter as an additional random effect, and none of the results is altered. A total of 58 people participated (65.5\% female; age (in years) $M=32.77, S D=14.8$ ).

127 Participants were tested individually in a single session in a curtained cubicle within a computer laboratory. The 128 experimenter withdrew from the cubicle during the tasks. On a desk in front of the participant was a desktop 129 computer with speakers and keyboard. Standard computer keyboards have slow polling rates that render them 130 unsuitable for tasks requiring highly accurate timing (Plant, Hammond \& Whitehouse, 2003; Verbruggen, Logan 
131 \& Stevens, 2008), and so we used a Razer 'Lycosa' games keyboard with a specified polling rate of $1000 \mathrm{~Hz}$. The 132 sequence of steps was as follows: participants read the study information sheet and signed a consent form; 133 completed a computerised demographic questionnaire and baseline mood measure (using the Qualtrics survey 134 platform, www.qualtrics.com); completed the mood induction task; completed the stop-signal task; and finally 135 completed another mood measure as a manipulation check. On completion, each participant was debriefed 136 and received the compensation payment.

137 Mood induction procedure. After completion of the demographic questionnaire, participants were 138 pseudorandomly assigned to either the negative or neutral conditions. This was done automatically by the 139 computer and the experimenter did not know which the experimental condition the participant was 140 completing. Participants in the negative condition were shown a sequence of 50 statements from the 141 depression Velten set (Velten, 1968), whereas participants in the neutral condition saw 50 from the neutral 142 set. Each statement was presented on the screen for six seconds, and the participants were instructed to try to 143 memorise them. After the mood induction had finished, the participant alerted the experimenter, who started 144 up the stop-signal task programme.

145 Stop signal task. We delivered the stop-signal task using STOP-IT software (Verbruggen, Logan \& Stevens, 146 2008). In this implementation, the go stimulus is a square or circle displayed in the middle of the screen; two 147 response keys on the keyboard were marked with a square and circle respectively, and the participant 148 instructed to press the correct one as quickly as possible. The stop signal is an audible tone. STOP-IT was run in 149 full-screen mode, with system volume set to full and speaker volume at $2 / 3$. The default task parameters were 150 used: 1 practice block of 32 trials, followed by 3 experimental blocks of 64 trials each. Each trial begins with a $151250 \mathrm{~ms}$ fixation cue, followed by the go stimulus, which is displayed until the participant responds, with a 152 maximum limit of $1250 \mathrm{~ms}$. The inter-trial interval is $2000 \mathrm{~ms}$. Stop-trials constitute $25 \%$ of all trials; the 153 difference in onset time of stop-cue relative to go-cue on these trials is automatically titrated dependent on 154 performance to provide an estimate of the delay at which that participant has a $50 \%$ chance of inhibiting 155 successfully. The participant's SSRT is calculated from the value of this delay.

156 After the experimenter had explained the instructions, participants pressed a key when they were ready to 157 start the task. The experimenter observed the participant during the practice block to see whether they were 158 responding correctly. The participant then completed the 3 experimental blocks, which the experimenter did 159 not observe. Following the practice block and each one of the trial blocks, a summary screen showing the 160 participant's response suppression rate, trials missed and errors made was displayed during a 10-second delay 161 until the participants could press a key to begin the next task 
163 Baseline and final mood. Baseline and final mood were rated with a single item, on a scale from 1 (the most 164 negative mood possible) to 100 (the most positive mood possible).

Neighbourhood deprivation score. We calculated a neighbourhood deprivation score using the postcode from age 16 provided by the participant. The age of 16 was chosen somewhat arbitrarily but seemed likely to be one the participant would remember as well as providing a reasonable summary measure of the kind of socioeconomic environment in which they had grown up. The UK Neighbourhood Statistics database (https://neighbourhood.statistics.gov.uk/) allows each UK postcode to be assigned a rank in terms of the deprivation of the census tract (lower super output area) in which it is found, from 1 (most deprived tract in

172 the UK) to 32482 (least deprived in the UK).To make these ranks more intuitively interpretable, we rescaled

173 them using the formula:

175 Thus, a postcode in the median UK neighbourhood would have a deprivation score of 0.5 , the most deprived a

176 score of 1 , and the least deprived a score of 0.

177 Age. Age was given in years in the initial demographic questionnaire.

178 SSRT and GRT. SSRT and GRT were calculated using the ANALYZE-IT programme supplied with STOP-IT

179 (Verbruggen, Logan \& Stevens, 2008). All participants provided data that met the assumptions required by

180 STOP-IT to generate a valid SSRT and GRT.

181 Data Analysis

182 Once the measures had been calculated, data were analysed in R (R Core Development Team, 2013), using

183 general linear models and t-tests as appropriate. The structure of each general linear model we ran is 184 described in the Results section. SSRTs were mildly right-skewed. All models reported below were also run 185 using log-transformed SSRT, which corrects the right skew. Since results were essentially identical, the non-

186 transformed results have been reported. Residuals were checked in all cases, and there were no major 187 violations of parametric model assumptions.

\section{RESULTS}

189 Raw data are available as Supplementary Material. 


\section{Descriptive statistics}

191 Table 1 shows the descriptive statistics by condition for variables other than performance in the stop-signal

192 task. There were no significant differences by condition in age, deprivation, or baseline mood, as there should 193 not have been, since condition was randomly assigned. Average deprivation scores were close to the UK 194 median neighbourhood (0.45), but with a good range $(0.11-0.98)$. Deprivation score was not significantly 195 associated with age $\left(r_{55}=0.18, p=0.18\right)$, or with baseline mood $\left(r_{56}=0.07, p=0.62\right)$.

\section{Mood manipulation and final mood}

197 The mood induction produced a modest decrease in mood between baseline and final rating in the negative condition $(M=-3.23 ; S D=5.27)$, and a modest improvement in mood in the neutral condition $(M=2.78 ; S D=$ 13.31). The condition difference in mood change was statistically significant $\left(t_{56}=-2.29, p=0.03\right)$. However, the final mood ratings were not significantly different between the negative and neutral conditions (Negative: $M=$ 77.1, $\mathrm{SD}=18.1$; Neutral: $\mathrm{M}=74.5, \mathrm{SD}=17.3, \mathrm{t}_{56}=0.56, \mathrm{p}=0.58$ ). This is due to variation in initial mood diluting the modest effect of the mood manipulation. For this reason, in all subsequent analyses, we include both experimental condition and baseline mood as independent variables. We also repeated the analyses presented below replacing experimental condition and baseline mood with final mood in the models. No effects of final mood were significant, and no conclusions were changed. Hence, these models are not described further.

\section{Go-reaction time (GRT)}

We fitted a general linear model to the GRTs, with age, initial mood, condition, deprivation score and the condition by deprivation score interaction as predictors. The model summary is shown in the upper part of table 2. Age predicted GRT, with GRTs becoming slower with increasing age, but there were no significant

211 effects of initial mood, condition or deprivation score on GRT.

\section{Stop-signal reaction time (SSRT)}

214 The general linear model for SSRT included as predictors GRT, age, initial mood, condition, deprivation score, 215 and the condition by deprivation score interaction. The model summary is shown in the lower part of table 2. 216 There was a significant effect of GRT, with participants who were faster on the go-trials having a longer SSRT. 217 There was also a predicted effect of age, with older participants having longer SSRTs. There was a significant 218 effect of deprivation score, with participants from more deprived neighbourhoods having longer SSRTs (figure 219 1). The effects of initial mood, condition, and the condition by deprivation interaction were not significant. 


\section{DISCUSSION}

223 In a community sample of adults, we found evidence that behavioural disinhibition, as measured using the 224 stop-signal task, was related to socioeconomic background. Participants who had lived in more deprived 225 neighbourhoods at age 16 had longer SSRTs, and hence showed greater behavioural disinhibition, than 226 participants who had lived in less deprived neighbourhoods. We also measured mood, and manipulated it 227 using a standard mood-induction procedure, but we found no evidence that current mood - either the naturally-occurring variation in baseline mood, or our experimentally-produced mood manipulation - affected stop-signal task performance in any way. Thus, our predictions concerning the relationship between socioeconomic deprivation and behavioural disinhibition were supported, whilst our predictions concerning

231 the role of current mood were not. Our results concurred with those of previous investigations in finding that 232 SSRTs, as well as GRTs, increased substantially with age (Williams et al., 1999; Bedard et al., 2010). We also 233 found that SSRT was negatively related to GRT; people who were faster to act in the go-trials were, other 234 things being equal, slightly more disinhibited. However, the deprivation-disinhibition relationship was not 235 explained by differences in GRT; it persisted even once variation in GRT was controlled for.

236 A number of previous studies have demonstrated socioeconomic gradients in measures of 'waiting' impulsivity, 237 the relatively steep devaluing of future rewards compared to immediate ones (Lawrance, 1991; Green et al., 238 1996; Adams \& White, 2009). To our knowledge, this is the first evidence that there may also be a socioeconomic gradient in 'stopping impulsivity' or behavioural disinhibition. Demonstrating the existence of 241 outcomes above and beyond 'waiting' impulsivity alone (Brevers et al., 2012; Sharma, Markon \& Clark, 2014).

242 Our results differ from those of the most comparable previous study (Crosbie et al., 2013), who found no 243 associations between socioeconomic deprivation and stop-signal performance in a large Canadian community 244 sample. Crosbie et al. used a similar approach to assessing deprivation as ours, using residential postcodes. 245 They report that their sample was skewed towards more economically privileged neighbourhoods (p. 501), 246 though direct comparison with the composition of our sample is not straightforward due to the differences in 247 measures and in countries. Crosbie et al. also studied children (mean age 11), whereas we studied adults.

248 Why socioeconomic gradients in impulsivity should exist is not well understood. One recent proposal is that 249 relationships between socioeconomic variables and impulsivity might be mediated by differences in mood 250 (Haushofer \& Fehr, 2014). However, this cannot be the case here, since there was no socioeconomic gradient 251 in baseline mood, and we found no evidence for any effect of mood on behavioural disinhibition. This is in 
contrast to a previous experimental study that found effects of mood manipulation on waiting impulsivity (Lerner, Li \& Weber, 2012). Thus, our data suggest an embedded effect of socioeconomic deprivation on disinhibition that is independent of current mood. Socioeconomic deprivation is associated with many different aspects of experience, and exactly what it is about deprivation that tends to produce greater disinhibition as well as a relatively stronger preference for immediate over delayed outcomes remains to be explored.

Our study had a number of limitations. Our sample was not constructed in such a way as to guarantee socioeconomic representativeness. However, we were fortunate in that the mean deprivation score of our sample was roughly in the middle of the UK range, and both extremes were represented in the data. We measured deprivation through postcode at age 16 . We chose this as we anticipated recruiting mainly young adults, and we felt this would be the single most convenient and effective measure for this age group. In fact, we recruited more, older people than anticipated, and childhood postcode at 16 is a less ideal measure for these participants than for younger ones. For one thing, their age 16 is longer ago, and the neighbourhoods may have changed in the intervening years. We made no attempt to distinguish statistically between deprivation experienced whilst growing up and adult deprivation. It could be that the most important predictor of behavioural disinhibition is current experience of deprivation (Nettle et al., 2014), and the relationship we found with deprivation at age 16 might be because people with more deprived childhood and adolescent backgrounds also go on to experience more deprivation in adulthood. To discriminate whether current or developmental experience of deprivation is important, the two would need to be separately measured to establish which has the greater predictive power. Our measure also did not distinguish individual-level socioeconomic characteristics such as parental income and education from neighbourhood-level deprivation. The two are likely to be highly correlated, but our study does not licence inferences about which of these, if either, is responsible for the observed association.

We did not measure current education level or any measure of general cognitive ability. This means we cannot exclude the possibility that general cognitive ability or education level mediates the relationship between socioeconomic background and behavioural disinhibition. There was no relationship between deprivation score and GRT, suggesting that the association was specifically with disinhibition, rather than with task performance more generally, but without a greater range of control tasks we are unable to make strong inferences on this point. More generally, caution is required about inferring causality from an association; though it seems reasonable that something about childhood deprivation might cause the development of greater disinhibition, other explanations for the correlation cannot be excluded. 
283 Our mood manipulation, though it employed a standard technique that has been used in other recent studies 284 (Smallwood \& O'Connor, 2011; Scherrer, Dobson \& Quigley, 2014), produced only very modest effects on 285 participants' final mood. It is possible that a stronger manipulation of mood might have affected behavioural 286 disinhibition. However, there was considerable variation in baseline mood, and the combination of baseline 287 mood and the manipulation still did not explain any variation in behavioural disinhibition. This suggests that 288 across a substantial range of mood, mood effects on behavioural disinhibition, if they exist, must be very small.

289 Despite the limitations noted above, it is striking that in a relatively small sample, and with a relatively crude 290 measure of socioeconomic background, we found evidence of an association between the experience of 291 deprivation and behavioural disinhibition. Childhood socioeconomic deprivation is an epidemiological predictor 292 of a number of important outcomes such as subsequent crime (Levine, 2011), and transition to habitual use of 293 drugs and tobacco (Legleye et al., 2011). Behavioural disinhibition plausibly plays a role in these outcomes.

294 Thus, behavioural disinhibition could be an important psychological mechanism linking childhood 295 socioeconomic conditions to subsequent life outcomes. 


\section{REFERENCES}

298

Adams J, White M. 2009. Time perspective in socioeconomic inequalities in smoking and body mass index. Health Psychology 28:83-90.

Bedard A-C, Nichols S, Jose A, Schachar R, Logan GD, Tannock R. 2010. The Development of Selective Inhibitory Control Across the Life Span. Developmental Neuropsychology 21:93-111.

Brevers D, Cleeremans A, Verbruggen F, Bechara A, Kornreich C, Verbanck P, Noël X. 2012. Impulsive Action but Not Impulsive Choice Determines Problem Gambling Severity. PLOS ONE 7:5-12.

Crosbie J, Arnold P, Paterson A, Swanson J, Dupuis A, Li X, Shan J, Goodale T, Tam C, Strug LJ, Schachar RJ. 2013. Response inhibition and ADHD traits: Correlates and heritability in a community sample. Journal of Abnormal Child Psychology 41:497-507.

Fillmore MT, Fillmore MT, Rush CR, Rush CR. 2002. Impaired inhibitory control of behavior in chronic cocaine users. Drug and alcohol dependence 66:265-73.

Green L, Myerson J, Lichtman D, Rosen S, Fry A. 1996. Temporal discounting in choice between delayed rewards: The role of age and income. Psychology and Aging 11:79-94.

Griskevicius V, Ackerman JM, Cantú SM, Delton AW, Robertson TE, Simpson JA, Thompson ME, Tybur JM. 2013. When the economy falters, do people spend or save? Responses to resource scarcity depend on childhood environments. Psychological Science 24:197-205.

Haushofer J, Fehr E. 2014. On the psychology of poverty. Science 344:862-867.

Kenealy PM. 1986. The Velten mood induction procedure: A methodological review. Motivation and Emotion 10:315-335.

Kidd C, Palmeri H, Aslin RN. 2013. Rational snacking: young children's decision-making on the marshmallow task is moderated by beliefs about environmental reliability. Cognition 126:109-14.

Kreek MJ, Nielsen D a, Butelman ER, LaForge KS. 2005. Genetic influences on impulsivity, risk taking, stress responsivity and vulnerability to drug abuse and addiction. Nature Neuroscience 8:1450-1457.

Lawrance EC. 1991. Poverty and the Rate of Time Preference: Evidence from Panel Data. Journal of Political Economy 99:54.

Legleye S, Janssen E, Beck F, Chau N, Khlat M. 2011. Social gradient in initiation and transition to daily use of tobacco and cannabis during adolescence: A retrospective cohort study. Addiction 106:1520-1531.

Lerner JS, Li Y, Weber EU. 2012. The Financial Costs of Sadness. Psychological Science 24:72-79.

Levine SZ. 2011. Elaboration on the association between IQ and parental SES with subsequent crime. Personality and Individual Differences 50:1233-1237. 
Logan GD, Cowan WB. 1984. On the ability to inhibit thought and action: A theory of an act of control. Psychological Review 91:295-327.

Logan GD, Cowan WB, Davis KA. 1984. On the ability to inhibit simple and choice reaction time responses: A model and a method. Journal of Experimental Psychology: Human Perception and Performance 10:276291.

Moffitt TE, Arseneault L, Belsky D, Dickson N, Hancox RJ, Harrington H, Houts R, Poulton R, Roberts BW, Ross S, Sears MR, Thomson WM, Caspi A. 2011. A gradient of childhood self-control predicts health, wealth, and public safety. Proceedings of the National Academy of Sciences of the United States of America 108:26932698.

Nettle D, Pepper G V, Jobling R, Schroeder KB. 2014. Being there : a brief visit to a neighbourhood induces the social attitudes of that neighbourhood. :1-20.

Perry JL, Carroll ME. 2008. The role of impulsive behavior in drug abuse. Psychopharmacology 200:1-26.

Pettersson E, Sjölander A, Almqvist C, Anckarsäter H, D’Onofrio BM, Lichtenstein P, Larsson H. 2014. Birth weight as an independent predictor of ADHD symptoms: A within-twin pair analysis. Journal of Child Psychology and Psychiatry and Allied Disciplines 4:453-459.

Plant RR, Hammond N, Whitehouse T. 2003. How choice of mouse may affect response timing in psychological studies. Behavior Research Methods, Instruments, \& Computers 35:276-284.

Pratt TC, Cullen FT. 2000. The empirical status of Gottfredson and Hirschi's general theory of crime: A metaanalysis. Criminology 38:931-964.

R Core Development Team. 2013. R: A Language and Environment for Statistical Computing.

Reynolds B, Ortengren A, Richards JB, de Wit H. 2006. Dimensions of impulsive behavior: Personality and behavioral measures. Personality and Individual Differences 40:305-315.

Sampson RJ, Sharkey P, Raudenbush SW. 2008. Durable effects of concentrated disadvantage on verbal ability among African-American children. Proceedings of the National Academy of Sciences of the United States of America 105:845-52.

Sastry N, Pebley AR. 2010. Family and neighborhood sources of socioeconomic inequality in children's achievement. Demography 47:777-800.

Scherrer MC, Dobson KS, Quigley L. 2014. Predictors of self-reported negative mood following a depressive mood induction procedure across previously depressed, currently anxious, and control individuals. The British journal of clinical psychology / the British Psychological Society 53:348-68.

Sharkey P, Elwert F. 2011. The legacy of disadvantage: multigenerational neighborhood effects on cognitive ability. American journal of Sociology 116:1934-81. 
360 Sharma L, Markon KE, Clark LA. 2014. Toward a theory of distinct types of "impulsive" behaviors: A meta361 analysis of self-report and behavioral measures. Psychological Bulletin 140:374-408.

362 Smallwood J, O'Connor RC. 2011. Imprisoned by the past: unhappy moods lead to a retrospective bias to mind 363 wandering. Cognition \& Emotion 25:1481-90.

364 Stahl C, Voss A, Schmitz F, Nuszbaum M, Tüscher O, Lieb K, Klauer KC. 2014. Behavioral components of 365 impulsivity. Journal of Experimental Psychology. General 143:850-86.

366 Velten E. 1968. A laboratory task for induction of mood states. Behaviour Research and Therapy 6:473-482.

367 Verbruggen F, Logan GD, Stevens M a. 2008. STOP-IT: Windows executable software for the stop-signal 368 paradigm. Behavior Research Methods 40:479-483.

369 Watson D, Tellegen A. 1985. Toward a consensual structure of mood. Psychological Bulletin 98:219-235.

370 Westermann R, Stahl G, Hesse FW. 1996. Relative effectiveness and validity of mood induction procedures : a 371 meta- analysis. European Journal of Social Psychology 26:557-580.

372

Williams BR, Ponesse JS, Schachar R, Logan GD, Tannock R. 1999. Development of inhibitory control across the life span. Developmental Psychology 35:205-213. 
Table $\mathbf{1}$ (on next page)

Descriptive statistics by condition for age, baseline mood and deprivation score. 
2

$\begin{array}{llll} & \text { Negative } & \text { Neutral } & \text { Condition difference } \\ \text { Participants } & 30 \text { (18 female }) & 28 \text { (20 female }) & \\ \text { Age (years) } & M=34.8, S D= & M=30.5, S D=13.3 & \mathrm{t}_{55}=1.09, \mathrm{p}=0.28 \\ & 15.9 & & \\ \text { Baseline Mood }(1- & M=80.4, S D= & M=71.7, S D=22.6 & \mathrm{t}_{56}=1.62, \mathrm{p}=0.11 \\ \text { 100) } & 17.9 & & \\ \text { Deprivation Score }(0- & M=0.45, S D= & M=0.45, S D=0.25 & \mathrm{t}_{55}=0.09, \mathrm{p}=0.93 \\ \text { 1) } & 0.29 & \end{array}$


Table 2 (on next page)

Summary of general linear models predicting go reaction time in the stop-signal task (GRT; upper rows) and stop-signal reaction time in the stop-signal task (SSRT; lower rows). 


\begin{tabular}{|c|c|c|c|c|}
\hline Variable & Parameter estimate & Standard error & $\mathrm{t}$ & $\mathrm{p}$ \\
\hline \multicolumn{5}{|c|}{ Outcome variable: GRT } \\
\hline Age & 4.85 & 1.35 & 3.59 & 0.01 \\
\hline Deprivation score & 185.20 & 225.82 & 0.82 & 0.42 \\
\hline Baseline mood & 0.46 & 0.98 & 0.47 & 0.64 \\
\hline Condition & 8.39 & 76.27 & 0.11 & 0.91 \\
\hline Deprivation score & -117.44 & 150.16 & -0.78 & 0.44 \\
\hline
\end{tabular}

Outcome variable: SSRT

$\begin{array}{lllll}\text { GRT } & -0.08 & 0.04 & -2.15 & 0.04 \\ \text { Age } & 1.08 & 0.41 & 2.63 & 0.01 \\ \text { Deprivation score } & 146.65 & 61.83 & 2.37 & 0.02 \\ \text { Baseline mood } & -0.40 & 0.27 & -1.50 & 0.14 \\ \text { Condition } & 18.40 & 20.75 & 0.89 & 0.38 \\ \text { Deprivation score * } & -65.26 & 41.09 & -1.59 & 0.12 \\ \text { condition } & & & & \end{array}$


Figure 1 (on next page)

Scatterplot of stop-signal reaction time (SSRT) against deprivation score, with participants labelled by condition in the mood manipulation. 


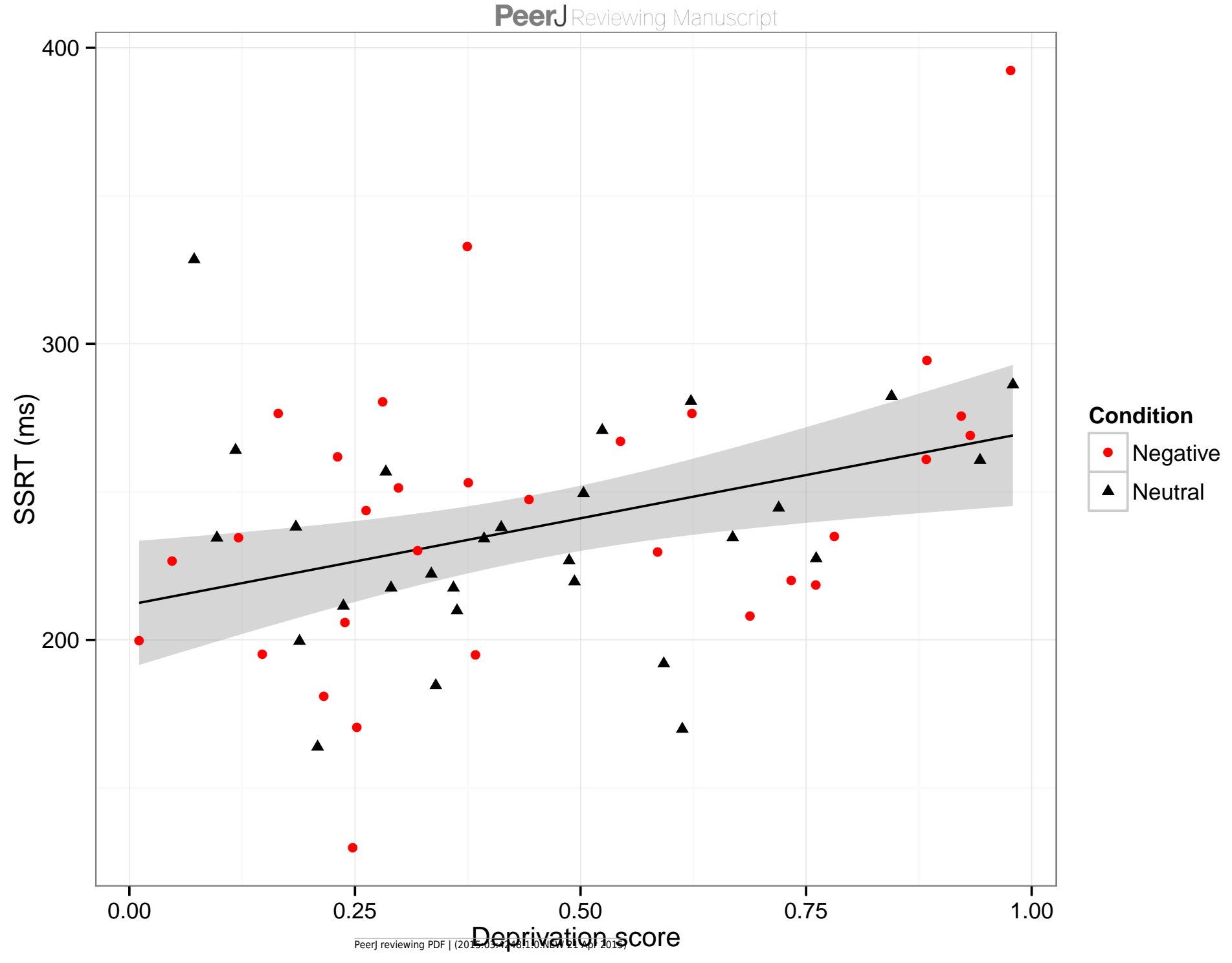

